\title{
Simulations of Cerebellar Motor Learning: Computational Analysis of Plasticity at the Mossy Fiber to Deep Nucleus Synapse
}

\author{
Javier F. Medina and Michael D. Mauk \\ W. M. Keck Center for the Neurobiology of Learning and Memory, and Department of Neurobiology and Anatomy, \\ University of Texas Medical School, Houston, Texas 77030
}

\begin{abstract}
We question the widely accepted assumption that a molecular mechanism for long-term expression of synaptic plasticity is sufficient to explain the persistence of memories. Instead, we show that learning and memory require that these cellular mechanisms be correctly integrated within the architecture of the neural circuit. To illustrate this general conclusion, our studies are based on the well characterized synaptic organization of the cerebellum and its relationship to a simple form of motor learning. Using computer simulations of cerebellarmediated eyelid conditioning, we examine the ability of three forms of plasticity at mossy fiber synapses in the cerebellar nucleus to contribute to learning and memory storage. Results suggest that when the simulation is exposed to reasonable
\end{abstract}

patterns of "background" cerebellar activity, only one of these three rules allows for the retention of memories. When plasticity at the mossy fiber synapse is controlled by nucleus or climbing fiber activity, the circuit is unable to retain memories because of interactions within the network that produce spontaneous drift of synaptic strength. In contrast, a plasticity rule controlled by the activity of the Purkinje cell allows for a memory trace that is resistant to ongoing activity in the circuit. These results suggest specific constraints for theories of cerebellar motor learning and have general implications regarding the mechanisms that may contribute to the persistence of memories.

Key words: LTP; LTD; cerebellum; eyelid conditioning; simulation; mossy fiber
Analysis of the neural basis of memory has been guided for some time by the central hypothesis that activity-dependent changes in synapses mediate changes in behavior. As an example that illustrates the importance of activity-dependent plasticity, consider Pavlov's classic learning experiments (Pavlov, 1927). With only those synapses activated by the bell eligible to be modified by the reinforcing meat powder, the learned salivation response would later be elicited relatively specifically when the bell is presented. For this reason, activity-dependent forms of plasticity, such as long-term potentiation (LTP) and long-term depression (LTD) in the hippocampus, neocortex and cerebellar cortex, have received particular attention (Siegelbaum and Kandel, 1991; Artola and Singer, 1993; Bear and Malenka, 1994; Linden, 1994).

Although learning requires that synaptic plasticity be limited to the right synapses (i.e., those activated by the bell), the capacity of memories to endure also requires that this new pattern of synaptic weights not be erased by the abundant opportunities for activity-dependent plasticity produced by ongoing brain activity (Sejnowski, 1977; Kenyon et al., 1998). Here we question a usually tacit hypothesis regarding the duration of memories: namely, that a molecular mechanism for persistent expression of synaptic plasticity is sufficient to explain the persistence of memories. With activity-dependent plasticity, this hypothesis involves the untenable assumption that synapses are only active during learning and are thus ineligible for plasticity at other times. Although applicable to many brain systems, we examine this issue with computer simulations of one type of cerebellar-mediated motor learning: Pavlovian conditioning of eyelid responses. Our results

\footnotetext{
Received March 16, 1999; revised May 7, 1999; accepted May 25, 1999.

This work was supported by National Institutes of Health Grant MH 57051.

Correspondence should be addressed to Dr. Michael D. Mauk, Department of Neurobiology and Anatomy, University of Texas Medical School, 6431 Fannin, Houston, TX 77030.

Copyright (C) 1999 Society for Neuroscience $\quad 0270-6474 / 99 / 197140-12 \$ 05.00 / 0$
}

illustrate the importance of considering how rules for plasticity must interact with neural circuits not only to permit the induction of plasticity during learning, but also to prevent the subsequent induction of unwanted plasticity despite ongoing brain activity.

Using the cerebellum as an example of a brain system involved in learning, we evaluate the ability of simulations to learn using plasticity in the cerebellar nucleus controlled by one of the three available signals. Evidence indicates that cerebellar-mediated motor learning, such as adaptation of the vestibulo-ocular response (VOR) or Pavlovian eyelid conditioning, involves plasticity at both granule to Purkinje cell $(g r \rightarrow P k j)$ synapses in the cerebellar cortex and mossy fiber to nucleus $(m f \rightarrow n u c)$ synapses in the cerebellar nuclei (Robinson, 1976; Perrett and Mauk, 1995; Raymond et al., 1996; Mauk, 1997) (see Fig. 1). Although both LTD and LTP have been identified and characterized in the cerebellar cortex (Sakurai, 1987; Ito, 1989; Hirano, 1990; Shibuki and Okada, 1992; Linden, 1994; Salin et al., 1996), nothing specific is known about the properties of the plasticity that occurs in the nucleus. Therefore, our cerebellar simulations incorporate the known climbing fiber-controlled (CF) form of plasticity at $g r \rightarrow P k j$ synapses and plasticity at $m f \rightarrow n u c$ synapses controlled by one of three possible cellular signals (see Fig. 2). These three signals are (1) the activity of the nucleus cell itself (Hebbian rule), (2) the activity of the climbing fiber input to the nucleus cell (climbing fiber-dependent rule), and (3) the activity of the Purkinje cell input to the nucleus (Purkinje-dependent rule). Results suggest that each of the three nucleus rules could support learning-with a distribution of plasticity between cortex and nucleus-if plasticity is permitted only during the training trials. In contrast, under the more realistic circumstance where the plasticity rule is applied at all times, nucleus and climbing fibercontrolled rules promote spontaneous drift of the strength of synapses during background inputs, precluding the possibility for learning or retention of responses. In addition to suggesting 


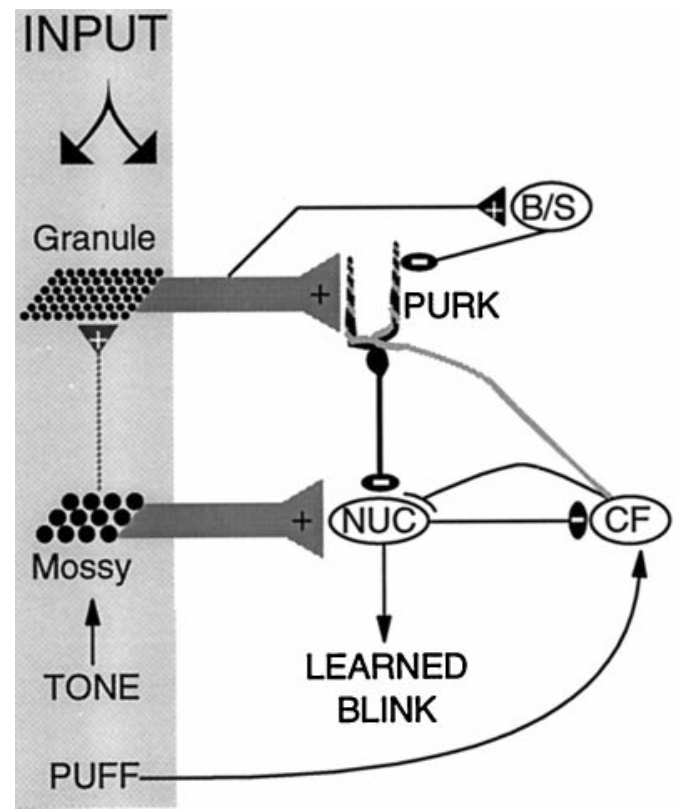

Figure 1. Simulations are based on the connectivity of the cerebellarolivary system and its relationship to eyelid conditioning. While the interpositus nucleus $(N U C)$ transmits the entire output of the cerebellum, two major excitatory afferents convey stimuli to the cerebellum. The mossy fiber afferent (Mossy) influences cerebellar output through direct excitatory connections onto the nuclei cells ( $m f \rightarrow n u c$ synapses) and through a more indirect projection onto a very large number of granule cells (Granule) that ultimately results in modulation of nuclei cells by Purkinje neurons (PURK). Granule cells affect Purkinje cell activity through connections to inhibitory interneurons known as basket and stellate cells $(B / S)$ and through a large number of direct excitatory synapses ( $g r \rightarrow P k j$ synapses) onto the Purkinje cell. In sharp contrast to this vastly diverging input, each Purkinje cell receives synaptic connections from a single climbing fiber $(C F)$, which also contacts the output cells of the cerebellar nuclei. Evidence indicates that conditioned stimuli such as tones are conveyed to the cerebellum via mossy fibers, the reinforcing air puff is conveyed via climbing fibers, and paired presentation of these stimuli leads to the expression of a conditioned eyelid response through increases in nucleus activity. This correspondence permits a simple representation of eyelid conditioning with a computer simulation of the cerebellum. Increases in simulated nucleus cell output during the conditioned stimulus are taken as a measure of the conditioned response. Presentation of the conditioned stimulus is emulated by altering the background activities of the mossy fibers and granule cells, whereas a transient excitatory input to the climbing fiber simulates the reinforcing puff. With these inputs determined, the remainder of the circuit is simulated with stochastic neurons (Materials and Methods). The simulations also implement the well characterized climbing fiber-dependent plasticity at the excitatory $g r \rightarrow P k j$ synapses and plasticity at the $m f \rightarrow n u c$ synapses controlled by one of three signals: nucleus cell activity, climbing fiber activity, or Purkinje cell activity.

specific constraints on the type of plasticity that may operate in the cerebellar nuclei during motor learning, these results have general implications regarding the mechanisms that may contribute to the persistence of memories.

\section{MATERIALS AND METHODS}

Network organization. The connectivity of the network is intended to capture the basic properties of the synaptic organization and physiology of the cerebellum (Eccles et al., 1967; Ito, 1984; Voogd and Glickstein, 1998) (see Fig. 1). Each of 20 Purkinje cells (PURK) receives, in addition to the CF input, inhibition from an average of 10 basket/stellate cells (B/S) and excitation from 200,000 granule cells (Granule). The sole output of the simulation is represented by a single nucleus cell (NUC) receiving a collateral climbing fiber input, inhibition from the Purkinje cells, and excitatory connections from 100 mossy fibers (Mossy). Consis- a) Cortex:Climbing fiber

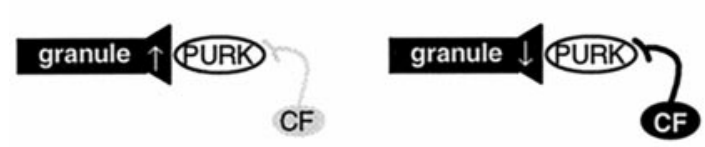

b1) Nucleus: Hebbian

mossy $\uparrow$ NUC mossy $\downarrow$ NUC

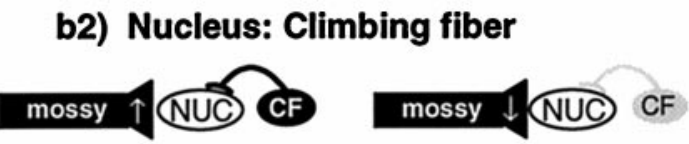

b3) Nucleus: Purkinje cell

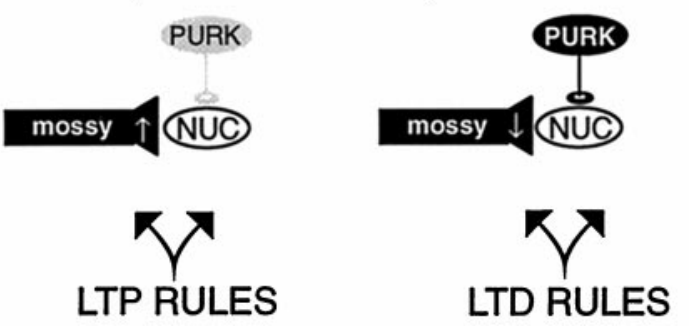

Figure 2. A representation of the well characterized, activity-dependent plasticity at the $g r \rightarrow P k j$ synapses in the cerebellar cortex, and three possible cellular rules for plasticity at $m f \rightarrow n u c$ synapses. Black symbols indicate that the cell is active, and gray symbols denote inactivity. Conditions for increasing the strength of synapses $(L T P)$ are shown in the left column, whereas the right column shows the signals that lead to the induction of $L T D$. a, Activity-dependent plasticity at $g r \rightarrow P k j$ synapses in the cerebellar cortex is controlled by climbing fiber inputs. These $g r \rightarrow P k j$ synapses undergo LTP when active in the absence of a climbing fiber input and undergo LTD when active in the presence of a climbing fiber input. $b 1$, With a Hebbian rule, active $m f \rightarrow n u c$ synapses undergo LTD when the nucleus cell is quiet and undergo LTP during periods of nucleus cell activity. $b 2$, With a climbing fiber-dependent rule, $m f \rightarrow n u c$ synapses that are active in the absence of a climbing fiber input to the nucleus cell undergo LTD and undergo LTP when the climbing fiber fires. b3, A Purkinje cell-dependent rule assumes that active $m f \rightarrow n u c$ synapses undergo LTD during periods of Purkinje inhibition of the nucleus and LTP during decreases in this inhibition.

tent with anatomical and physiological observations, the circuit was modeled as a closed loop in which the Purkinje cells inhibit a nucleus cell that inhibits the climbing fiber that provides input to the Purkinje cells (Voogd and Bigare, 1980; Buisseret-Delmas and Angaut, 1993; Ruigrok, 1997; Miall et al., 1998; Voogd and Glickstein, 1998). The number of presynaptic inputs received by each simulated cell is summarized in Table 1.

Representation of neural activity. Synaptic transmission in the nervous system is a noisy process brought on by random fluctuations in the release of neurotransmitter and other probabilistic causes. We have captured this inherent noisiness by implementing the traditional method for introducing a stochastic mechanism in the firing of neurons (Haykin, 1994). Specifically, the probability of a neuron's firing is calculated each simulated time bin and can be approximated by a simple, sigmoid function of the membrane potential, $V$, according to the equation:

$$
P(V)=\frac{1}{1+e^{-(V-\theta)}} .
$$


Table 1. Parameters related to the activity of the simulated cells

\begin{tabular}{|c|c|c|c|}
\hline & $\begin{array}{l}\text { Number per (post- } \\
\text { synaptic target) }\end{array}$ & Background activity & Activity representation \\
\hline \multicolumn{4}{|l|}{ Input cells ${ }^{a}$} \\
\hline Mossy fibers & 100 (nucleus) & Constant $(\sim 50 \mathrm{~Hz})$ & $\begin{array}{l}\text { Gaussian distribution with a } 0 . \\
\text { mean within the interval }[0,1]\end{array}$ \\
\hline Granule cells & $\begin{array}{c}200,000 \text { (Purkinje) } \\
\text { 2,000 (basket) }\end{array}$ & Constant $(\sim 50 \mathrm{~Hz})$ & $\begin{array}{l}\text { Gaussian distribution with a } 0 . \\
\text { mean within the interval }[0,1]\end{array}$ \\
\hline \multicolumn{4}{|l|}{ Other cells ${ }^{b}$} \\
\hline Basket cells & 10 (Purkinje) & $\sim 20 \mathrm{~Hz}$ & $P(V)=\frac{1}{1+e^{-(V-\theta)}}, \theta=7.2$ \\
\hline Purkinje cells & 20 (nucleus) & $\sim 80 \mathrm{~Hz}$ & $P(V)=\frac{1}{1+e^{-(V-\theta)}}, \theta=5.3$ \\
\hline Nucleus cells & 1 (climbing fiber) & $\sim 40 \mathrm{~Hz}$ & $P(V)=\frac{1}{1+e^{-(V-\theta)}}, \theta=6.0$ \\
\hline Climbing fiber & 1 (Purkinje) & $\sim 1 \mathrm{~Hz}$ & $P(V)=\frac{1}{1+e^{-(V-\theta)}}, \theta=3.3$ \\
\hline
\end{tabular}

These parameters were the same in all simulations, independent of the plasticity rule implemented at the mossy fiber synapse.

${ }^{a}$ Probability of activity chosen from a Gaussian distribution.

${ }^{b}$ Probability of activity calculated based on strength of presynaptic inputs.

This standard function mimics the biological relationship between the cell's input current and its firing rate in several ways: the output is always non-negative, it is very small below the cell's threshold, $\theta$ (see Table 1 for the values implemented in the different cells that were simulated), it monotonically increases with input, and it has an upper bound at 1 . Whether a cell fires (where the term "fires" can be applied to a single spike or alternatively a burst of spikes) is determined each simulated time bin by comparing the newly calculated probability with a newly generated random number taken from a uniform distribution in the interval $[0,1]$. Thus:

$$
\begin{aligned}
& \text { if } P(V) \geq \text { random number, Spike }=1, \\
& \text { if } P(V)<\text { random number, Spike }=0 .
\end{aligned}
$$

The general description given in the previous paragraph of how activity is calculated for each of the cells that participate in the simulation (excluding mossy fibers and granule cells; see below) can be formalized with the following equations. The membrane potential of each basket/ stellate cell is calculated by adding the synaptic weights of active presynaptic granule cell inputs:

$$
V_{b / s}=\frac{\sum_{i=1}^{l} \operatorname{Spike}_{g r}^{i} w_{g r}^{i}}{l},
$$

where $l$ is typically set to 2000 (i.e., the number of granule synapses made onto each basket/stellate cell). The activity of each of these cells is then obtained by applying the described sigmoidal function:

$$
P_{b / s}=\frac{1}{1+e^{-\left(V_{b / s}-\theta_{b / s}\right)}},
$$

with $\theta_{b / s}$ set to 7.2 to allow basket/stellate cells to discharge at their physiological spontaneous rate of $\sim 20 \mathrm{~Hz}$ (Armstrong and Rawson, 1979), which corresponds to a 0.1 value for $P_{b / s}$ when using a $5 \mathrm{msec}$ time-step. Interestingly, the particular discharge rate of these or any of the other cells were found to have no effect on the general validity of the results because they affected all plasticity rules equally.

The membrane potential of each Purkinje cell is given by:

$$
V_{p k j}=\frac{\sum_{i=1}^{m} S_{p i k e} e_{g r} w_{g r}^{i}}{m}-\frac{\sum_{i=1}^{k} P_{b / s}}{k},
$$

where the first term sums the weights of active granule cells and the other term represents inhibition from active basket/stellate cells. $m$ and $k$ are the number of granule and basket/stellate cell inputs to the Purkinje cell.
The activity of each Purkinje cell is always obtained by applying a threshold function (sigmoidal) to the neuron's membrane potential except when its associated climbing fiber fires. The empirically observed pause in Purkinje cell activity after a climbing fiber input is simulated by momentarily setting the probability of activity of the Purkinje cell to 0 :

$$
\begin{aligned}
& \text { if climbing fiber spike, } P_{p k j}=0, \\
& \text { otherwise, } P_{p k j}=\frac{1}{1+e^{-\left(V_{p k j}-\theta_{p k j)}\right.}},
\end{aligned}
$$

with $\theta_{p k j}$ set to 5.3 to allow Purkinje cells to discharge at their physiological spontaneous rate of $\sim 80 \mathrm{~Hz}$ (Thach, 1968), which corresponds to a 0.4 value for $P_{p k j}$ when using a $5 \mathrm{msec}$ time-step. This variable represents the total inhibitory effect of each Purkinje cell on nucleus activity.

Similarly, the membrane potential for the nucleus cell is given by:

$$
V_{n u c}=\frac{\sum_{i=1}^{n} S_{p i k e_{m f}^{i} w_{m f}^{i}}^{i}}{n}-\frac{\sum_{i=1}^{j} P_{p k j}}{j}+P_{c f},
$$

where the first term represents excitation of the nucleus cell via active mossy fibers, the second represents inhibition from the Purkinje cells, and the third represents excitation from the climbing fiber. $j$ and $n$ are the total number of Purkinje cell and mossy fiber inputs to the nucleus cell. The activity of the nucleus cell is then obtained by applying a threshold function (sigmoidal) to the neuron's membrane potential:

$$
P_{n u c}=\frac{1}{1+e^{-\left(V_{n u c}-\theta_{n u c}\right)}},
$$

with $\theta_{\text {nuc }}$ set to 6.0 to allow the nucleus cell to discharge at its physiological spontaneous rate of $\sim 40 \mathrm{~Hz}$ (Thach, 1968), which corresponds to a 0.2 value for $P_{n u c}$ when using a $5 \mathrm{msec}$ time-step. This variable represents the total inhibitory effect of the nucleus cell on climbing fiber activity.

Finally, the membrane potential for the climbing fiber is given by:

$$
V_{c f}=E_{u s}-K_{n u c} P_{n u c},
$$

where $E_{u s}$ represents the excitatory effect of a possible unconditioned stimulus (US) and the last term represents the inhibitory action of the nucleus cell on climbing fibers $\left(K_{\text {nuc }}\right.$ is typically set to 10 so that $V_{c f}$ can range from -10 to 0 in the absence of a US although $P_{n u c}$ ranges only from 0 to 1 ). The activity of the climbing fiber is then obtained by applying a threshold function (sigmoidal) to its membrane potential:

$$
P_{c f}=\frac{1}{1+e^{-\left(V_{c f}-\theta_{c f}\right)}} \text {, }
$$


with $\theta_{c f}$ set to 3.3 to allow the climbing fiber to discharge at its physiological spontaneous rate of $\sim 1 \mathrm{~Hz}$ (Keating and Thach, 1995), which corresponds to a 0.005 value for $P_{c f}$ when using a $5 \mathrm{msec}$ time-step.

The probabilities of activity for the input elements (i.e., granule cells and mossy fibers) were specified and remained constant for each simulation (Table 1). Thus, the excitatory connection between mossy fibers and granule cells was not explicitly simulated, but rather the probabilities of activity for these cells were independently chosen from separate Gaussian distributions in the interval $[0,1]$. This implementation allowed for independent control of the mean level of activity of these inputs. For all the plasticity rules examined, the rate at which $g r \rightarrow P k j$ and $m f \rightarrow n u c$ synapses changed increased with higher levels of granule cell and mossy fiber activity, respectively (data not shown). However, as long as the mean activity was kept constant when comparing different plasticity rules, the conclusions of this study did not depend on a particular choice for input activity. Typically, the mean levels of activity for both inputs were $\sim 50 \mathrm{~Hz}$ (Eccles et al., 1971) (i.e., the mean of the Gaussian distributions was 0.25 with a $5 \mathrm{msec}$ time step). In contrast to the way the activities for inputs were specified, the activities of basket/stellate cells, Purkinje cells, the nucleus cell, and the climbing fiber were calculated for each time step based on the strength of their presynaptic inputs. For these cells, activity was determined by summing the weights of all active excitatory and inhibitory synapses for a time bin and calculating a probability of activity from these inputs.

Plasticity rules at modifiable synapses. The key assumption of our simulations is that two sets of synapses in the cerebellum can undergo changes in strength during motor learning. On the basis of evidence that supports this assumption (Robinson, 1976; Perrett and Mauk, 1995; Raymond et al., 1996; Mauk, 1997), we have implemented a plasticity rule that specifies that $g r \rightarrow P k j$ synapses decrease in strength when active in the presence of a climbing fiber input and increase in strength when active in the absence of a climbing fiber input (Sakurai, 1987; Ito, 1989; Hirano, 1990; Shibuki and Okada, 1992; Linden, 1994; Salin et al., 1996). Although these studies clearly illustrate the dependence of LTD/LTP at $g r \rightarrow P k j$ synapses on climbing fiber activity, there is a lack of evidence to support the model's assumption that LTD and LTP can reverse each other. In fact, the evidence to date suggests that LTP/LTD at this synapse is not bidirectional because the expression of the former seems to be presynaptic (Salin et al., 1996), whereas that of the later is clearly postsynaptic (Linden, 1994). However, the fact that this reversal is a feature common to various CNS synapses, including hippocampus (Heynen et al., 1996), visual cortex (Kirkwood et al., 1993), motor cortex (Hess and Donoghue, 1996), and inferotemporal cortex (Chen et al., 1996), highlights the plausibility that $g r \rightarrow P k j$ synapses could also display the same property.

Given known anatomical constraints (Eccles et al., 1967; Ito, 1984), we have also implemented bidirectional plasticity at $m f \rightarrow n u c$ synapses controlled by activity in one of the inputs to the nucleus cell (i.e., climbing fiber or Purkinje cells) or in the nucleus cell itself (see Fig. 2). The presence of plasticity at $m f \rightarrow n u c$ synapses is at present an assumption of the model. Although these synapses contain NMDA receptors (Cull-Candy et al., 1998), there is only one report of plasticity at this location, and even then the induction protocol required nonphysiological stimulating conditions (Racine et al., 1986). Mathematically, the changes in strength at $g r \rightarrow P k j$ and $m f \rightarrow n u c$ synapses are implemented as follows:

(1) Plasticity at $g r \rightarrow P k j$ synapses:

$$
\Delta w_{i}^{g r}=\frac{\delta_{-}^{g r} \cdot S p i k e_{i}^{g r} \cdot S p i k e^{c f}}{L T D}+\frac{\delta_{+}^{g r} \cdot S p i k e_{i}^{g r} \cdot\left(1-S_{p i k e^{c f}}\right)}{L T P} ;
$$

(2) plasticity at $m f \rightarrow n u c$ synapses:

$$
\begin{gathered}
\Delta w_{i}^{m f}=\frac{\delta_{-}^{m f} \cdot \text { Spike }_{i}^{m f} \cdot\left(1-\text { Spike }^{n u c}\right)}{L T D} \\
+\frac{\delta_{+}^{m f} \cdot{\text { Spike } e_{i}^{m f} \cdot S p i k e^{n u c}}_{L T P}}{L} \quad \text { (Hebbian rule) } \\
\Delta w_{i}^{m f}=\frac{\delta_{-}^{m f} \cdot S p i k e_{i}^{m f} \cdot\left(1-S_{\left.p i k e^{c f}\right)}\right.}{L T D} \quad \text { (climbing fiber rule) }
\end{gathered}
$$

$$
\begin{aligned}
\Delta w_{i}^{m f}=\frac{\delta_{-}^{m f} \cdot}{S p i k e_{i}^{m f} \cdot\left(\text { Spike }^{P k j}\right)} & \text { LTD } \\
& +\frac{\delta_{+}^{m f} \cdot \text { Spike }_{i}^{m f} \cdot\left(1-\text { Spike }^{P k j}\right)}{L T P}, \quad \text { (Purkinje cell rule) }
\end{aligned}
$$

where the different Spike terms are calculated as shown in Equations 2 and 3 , and $\delta_{+}^{g r}=0.001, \delta_{-}^{g r}=-0.199, \delta_{+}^{m f}=0.001$, and $\delta_{-}^{m f}=-0.0015$ are constants that represent step decreases or increases in the strengths of $g r \rightarrow P k j$ and $m f \rightarrow n u c$ synapses that were kept the same for all simulations independent of the plasticity rule implemented at the mossy fiber synapse. Under this mathematical representation, the ith $g r \rightarrow P k j$ or $m f \rightarrow n u c$ synapse is modified every time it is active (i.e., when $S p i k e_{i}^{g r}$ or Spike $_{i}^{m f}$ equals 1) and will undergo LTP or LTD depending on whether the Spike term of the signal controlling plasticity equals 0 or 1 . However, the results presented here did not change when these plasticity rules were modified to allow a range of frequencies in the plasticity-controlling signals that left synaptic strengths unchanged.

The precise relative timing between the Spike variables controlling synaptic changes in the implementation of the plasticity rules outlined above deserves further consideration. For example, the rule for LTD at $g r \rightarrow P k j$ synapses results in a decrease of the strength of synapses that are active simultaneously with a climbing fiber input. Although it is true that only sharp temporal relations between these signals have been found to promote LTD in the Purkinje cells of the electric fish Gnathonemus petersii (Bell et al., 1997), this is not so in most cases. The lack of temporal constraints in protocols capable of inducing cerebellar longterm depression is apparent in various studies in which effective timing relations between presynaptic activation of $g r \rightarrow P k j$ synapses and climbing fiber inputs have ranged from presynaptic first by $250 \mathrm{msec}$ (Chen and Thompson, 1995) to climbing fiber first by $125 \mathrm{msec}$ (Ekerot and Kano, 1989). Our choice of a $0 \mathrm{msec}$ interval (i.e., simultaneous activity in $g r \rightarrow P k j$ synapse and climbing fiber input) represents a consensus interval that seems to allow induction of plasticity under various experimental conditions (Ekerot and Kano, 1989; Karachot et al., 1994; Chen and Thompson, 1995).

Simulating eyelid conditioning. During Pavlovian conditioning, the presentation of an initially neutral conditioned stimulus (CS) is paired with a reinforcing US. After repeated CS + US pairings, the CS acquires the ability to elicit a conditioned response. In the case of eyelid conditioning, paired presentation of tone-CS and puff-US results in a conditioned eyelid closure elicited by the tone (Schneiderman et al., 1962). Converging evidence from a number of laboratories suggests that the tone is conveyed to the cerebellum by mossy fibers (Steinmetz et al., 1985, 1986, 1987, 1988; Solomon et al., 1986; Lewis et al., 1987) the puff is conveyed by climbing fibers (McCormick et al., 1985; Mauk et al., 1986), and that increases in the activity of cerebellar output cells in the anterior interpositus nucleus drive the expression of eyelid response (McCormick and Thompson, 1984).

Given the straightforward manner in which these stimuli map onto the afferent pathways to the cerebellum, eyelid conditioning can be relatively easily represented in our simulations. Adding a constant (Eus) to the firing probability of the climbing fiber simulates the presence of the US during acquisition trials. This constant was chosen so that initially, when the US is presented, the probability of activity of the climbing fiber is 1 . The CS is represented by changing the probability of firing of the ith granule cell from $P_{g r}^{i}$ to $P C S_{g r}^{i}$ and that of the ith mossy fiber from $P_{m f}^{i}$ to $P C S_{m f}^{i}$ for the duration of the stimulus. Although the conclusions of the paper with respect to the stability of plasticity rules did not depend on these particular activities, our results suggest that in general, learning occurred faster as PCS became more different from $P$ (data not shown). For the simulations shown, separate probabilities for mossy fibers and granule cells were assigned from Gaussian distributions with a mean of 0.25 and a variance of 0.20 such that $0 \leq P_{g r}^{i}, P C S_{g r}^{i}, P_{m f}^{i}, P C S_{m f}^{i} \leq 1$. With a 5 msec time-step, the 0.25 mean represents a discharge rate of 50 $\mathrm{Hz}$ for these inputs.

Timing of eyelid conditioning stimuli. Models of classical conditioning have been traditionally divided into three broad categories (Gluck et al., 1990): trials-level, temporal, and real-time models. Trials-level models treat the CS as a unitary event and consider only the net effects of each trial. Temporal models include factors that address the limited range of interstimulus intervals that promote conditioning (the ISI function), and real-time models also address the timing or temporal properties of conditioned responses. In this respect, the present simulations represent a trials-level analysis because we did not attempt to capture the sensitiv- 
ity that exists to the temporal relationships between CS and US. Therefore, our simulations can only describe the net effects of a training trial on the strength of CS-US associations by presenting simultaneous CS-US pairings lasting a single time-step.

Types of simulations. The simulations were of two general forms. During "conditioning simulations," inputs corresponded to the presentation of stimuli during Pavlovian conditioning as described above without any activity between learning trials. In contrast, "background simulations" included background activity between occasional presentations of the $\mathrm{CS}$. However, the strengths of $g r \rightarrow P k j$ and $m f \rightarrow n u c$ synapses were not allowed to change during these occasional CS presentations, which were necessary only to assess the retention of a memory previously formed by a conditioning simulation.

\section{RESULTS}

\section{Acquisition of conditioned eyelid responses}

The architecture and events of the simulations were based on the well characterized synaptic organization of the cerebellum (Eccles et al., 1967; Ito, 1984; Voogd and Glickstein, 1998) and its relationship to Pavlovian eyelid conditioning (Thompson, 1986; Thompson and Krupa, 1994; Mauk and Donegan, 1997) (Fig. 1). Eyelid conditioning involves the paired presentation of a CS such as a tone and a reinforcing US such as a puff of air directed at the eye. With repeated trials, the CS acquires the ability to elicit conditioned closure of the eyelid (Schneiderman et al., 1962). Previous studies have demonstrated that information about the CS and US is conveyed to the cerebellum via mossy fiber (Steinmetz et al., 1985, 1986, 1987, 1988; Solomon et al., 1986; Lewis et al., 1987) and climbing fiber inputs (McCormick et al., 1985; Mauk et al., 1986), respectively, and that output of the cerebellum via the interpositus nucleus is necessary for the expression of the conditioned responses (McCormick and Thompson, 1984). It is this wealth of knowledge about the synaptic organization of the cerebellum, its relationship to eyelid conditioning, and sites and mechanisms for plasticity that make this brain system an ideal structure with which to study interactions between network properties and forms of plasticity. Furthermore, the correspondence between eyelid conditioning and cerebellar inputs and outputs permits a relatively straightforward representation with simulations. Presentation of the CS is simulated by briefly altering the background activities of the mossy fiber (and granule cell) inputs, and presentation of the US is simulated by applying a transient excitatory input to the climbing fiber. Increases in simulated nucleus cell output during the CS are then taken as an index of conditioning. These eyelid conditioning trials were presented in three separate simulations that incorporated the well characterized climbing fiber-dependent plasticity at $g r \rightarrow P k j$ synapses and one of three possible plasticity rules at $m f \rightarrow n u c$ synapses (Fig. 2).

Although under the conditions illustrated in Fig. 2 a $m f \rightarrow n u c$ synapse is modified every time it is active, our results do not depend on this simplification. We also implemented plasticity under the control of signals related to rates of activity rather than single spikes. In this case, it is possible to specify a range of activity frequencies that will not modify the strength of active synapses. For example, a Hebbian rule could induce LTP when the nucleus cell is firing at a high frequency, induce LTD at low nucleus cell frequencies, and leave synaptic strengths unchanged when the nucleus is firing at intermediate frequencies. The nature of the results did not depend on these different implementations as long as the frequencies that induced plasticity could be attained, thus providing opportunities for synaptic modification.

Initially, learning was simulated with the standard modeling practice of permitting plasticity only during the presentation of

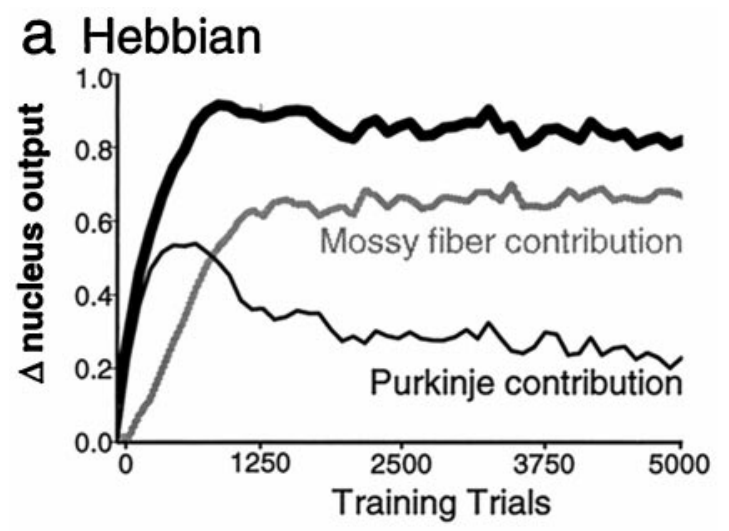

b Climbing fiber-dependent

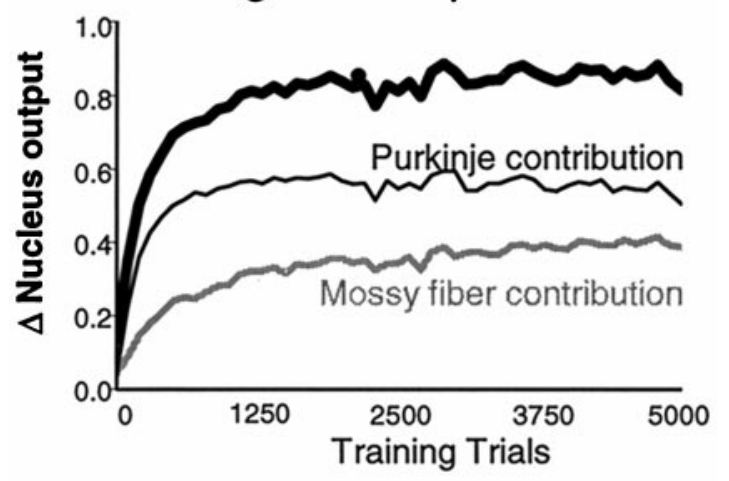

C Purkinje cell-dependent

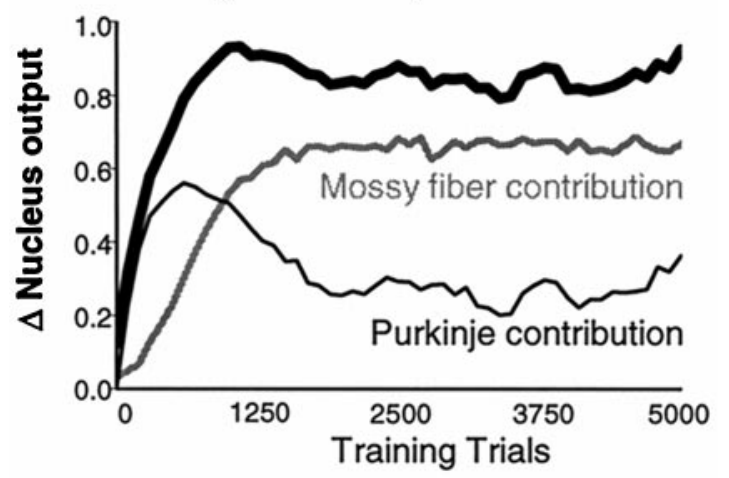

Figure 3. Acquisition of simulated conditioned responses when a Hebbian $(a)$, climbing fiber-dependent $(b)$, or Purkinje-dependent $(c)$ plasticity rule is implemented at $m f \rightarrow n u c$ synapses and plasticity is permitted only during the training trial. Increases in nucleus cell activity during presentation of the CS (thick black line) provide a measure of the conditioned response amplitude. The contributions made to the conditioned response by plasticity at $g r \rightarrow P k j$ synapses in the cerebellar cortex (thin black line) and by plasticity at $m f \rightarrow n u c$ synapses in the cerebellar nucleus (gray line) are also shown. Consistent with existing data from eyelid conditioning and VOR adaptation, conditioned responses were produced by a combination of plasticity at these two sites.

the conditioning trial (Fujita, 1982; Moore et al., 1989; Gluck et al., 1990; Fiala et al., 1996) and not at other times. Under these conditions, all three simulations acquired conditioned responses; as training proceeded, they showed increases in nucleus cell output during the simulated CS (Fig. 3). Moreover, these condi- 
tioned responses were produced by a combination of plasticity in both the cerebellar cortex and cerebellar nucleus, which is consistent with results from previous studies on eyelid conditioning and VOR adaptation (Robinson, 1976; Perrett et al., 1993; Raymond et al., 1996; Mauk, 1997). For all three simulations, presentation of mossy fiber/granule cell inputs paired with a climbing fiber input led to the induction of LTD at the CS-activated $g r \rightarrow P k j$ synapses. As training proceeded, this resulted in a learned decrease in Purkinje cell activity during the CS input (Fig. 3, Purkinje contribution). Although the details differ slightly for the three rules, training also led to the induction of LTP at the $m f \rightarrow n u c$ synapses (Fig. 3, Mossy fiber contribution). For Hebbian and Purkinje-dependent rules, the acquired decrease in Purkinje activity during the CS aids in the induction of LTP at the $m f \rightarrow n u c$ synapses either directly (i.e., Purkinje-dependent rule) or by disinhibiting the nucleus (i.e., Hebbian rule). For the climbing fiber-dependent rule, the induction of LTP at $m f \rightarrow n u c$ synapses is a direct consequence of the activation of the climbing fiber during the US and proceeds independently of the activity of the Purkinje cell during training. Thus, for the three simulations, LTD in the cerebellar cortex reduced the strength of the inhibitory action of Purkinje cells onto the nucleus, whereas LTP of mossy fibers increased the strength of the excitatory input to the nucleus. Both of these changes contributed to the expression of the conditioned response by increasing the probability of firing of the nucleus cell. When the CS was presented in the absence of the US, all three simulations produced similar extinction of the conditioned response by reversing the changes that had occurred during acquisition (data not shown).

\section{Retention of conditioned eyelid responses}

Stopping here might lead to the conclusion that a synaptic mechanism for the long-lasting expression of LTD and LTP at the $g r \rightarrow P k j$ and $m f \rightarrow n u c$ synapses would ensure the persistence of this memory for motor learning. Simply continuing the simulation with a low level of background input activity and with the plasticity rules operational shows that this is not necessarily true. As shown in Figure 4, two of the nucleus plasticity rules, Hebbian (dark gray line) and climbing fiber-dependent (light gray line), produced a spontaneous drift in the strengths of the $g r \rightarrow P k j$ and $m f \rightarrow n u c$ synapses. This rapidly caused synapses to saturate at their maximum or minimum possible values, erasing the learned pattern of synaptic weights, and thus abolishing the previously learned response. Although specific parameters determine whether the synapses drift upward and saturate at maximum values or downward to minimum values, the tendency to drift itself is parameter independent and occurs for all non-zero forms of background activity.

In contrast to this rapid loss of memory, responses were retained orders of magnitude longer when the simulations implemented plasticity at $m f \rightarrow n u c$ synapses that was directly controlled by Purkinje cell activity (Fig. 4, black line). As shown in the top graph of Figure 5, in these simulations the activity of the signal controlling plasticity at $g r \rightarrow P k j$ synapses (i.e., climbing fiber activity; thick black line) was self-regulated to an equilibrium level where $g r \rightarrow P k j$ synapses were as likely to decrease in strength when active during a climbing fiber input as they were to increase in strength when active in its absence. Similarly, the activity of the signal controlling plasticity at $m f \rightarrow n u c$ synapses (i.e., Purkinje cell activity; thin black line) was maintained at an equilibrium level that balanced LTP and LTD at $m f \rightarrow n u c$ synapses such that although the strength of a synapse was modified every time it was active, the net change was zero (Fig. 5, bottom graph). When the system is at this equilibrium state, synapses can still experience LTD and LTP events, thus essentially performing a random walk that eventually erases memories. The key is that memories last much longer because this equilibrium state (see Appendix for a formal mathematical analysis of the conditions that lead to this equilibrium state) does not promote the directed drift toward maximum or minimum synaptic values observed when unstable rules (i.e., Hebbian and climbing fiber dependent) were implemented at $m f \rightarrow n u c$ synapses. Furthermore, the properties that allow the Purkinje-dependent rule to maintain synaptic strength do not preclude the extinction of conditioned responses when the CS is presented in the absence of the US.

\section{Differences between stable and unstable rules}

The fundamental differences between simulations that incorporate the two unstable rules (Hebbian and climbing fiber dependent) and those that implement a rule that is directly controlled by the level of Purkinje cell activity are illustrated in Figures 5 and 6. A Purkinje-dependent rule for $m f \rightarrow n u c$ synapses allows for independent regulation of plasticity at $g r \rightarrow P k j$ synapses controlled by the level of climbing fiber activity and at $m f \rightarrow n u c$ synapses controlled by the level of Purkinje cell activity. The ability of the simulated cerebellar network to independently regulate climbing fiber and Purkinje cell activities is apparent from the synaptic organization of the cerebellum. As shown in Figure 1 , in addition to the climbing fiber input, Purkinje cells also receive a modifiable input from granule cells (the $g r \rightarrow P k j$ synapses). The consequence is that although climbing fiber activity affects the activity of the Purkinje cell, it does not determine it. Figure 1 also illustrates that Purkinje cell activity, by itself, does not determine the activity of its associated climbing fiber. Although the nucleus cell functionally connects Purkinje cells to the climbing fiber, the activity of the nucleus cell is modulated by its modifiable $m f \rightarrow n u c$ input. As a consequence, the activity of the nucleus cell (and thus the climbing fiber) is affected but not determined by the activity of its Purkinje cell input. Thus, it is possible for a level of Purkinje cell activity suitable for stability of $m f \rightarrow n u c$ synapses to co-exist with a level of climbing fiber activity suitable for stability of $g r \rightarrow P k j$ synapses.

The key difference that prevents stability of synaptic strength with Hebbian and climbing fiber-dependent rules is in each case a strong coupling of the signals that control plasticity at both modifiable sites. This coupling means that simultaneous stability of both $m f \rightarrow n u c$ and $g r \rightarrow P k j$ synapses can be achieved only under a very specific set of parameters (see Appendix for a formal mathematical analysis). In the case of simulations that implement a climbing fiber-dependent rule (Fig. 6), changes at $m f \rightarrow n u c$ and $g r \rightarrow P k j$ synapses are coupled because the signals controlling plasticity (i.e., climbing fiber activity; thick black line) are identical at both sites. The situation is similar when a Hebbian rule is implemented at $m f \rightarrow n u c$ synapses because the signal controlling plasticity at $g r \rightarrow P k j$ synapses (i.e., climbing fiber activity) is always completely determined by the signal controlling plasticity at $m f \rightarrow n u c$ synapses (i.e., nucleus cell activity). Therefore, for these two cases there are two sets of modifiable synapses under the control of a single signal related to the activity of the climbing fiber. Unless a specific set of parameters is carefully chosen so that both $m f \rightarrow n u c$ and $g r \rightarrow P k j$ synaptic strengths can be maintained constant by the same level of climbing fiber activity, they will spontaneously drift, erasing any previously formed memory (Fig. 6, bottom right). This situation is analogous to controlling the 

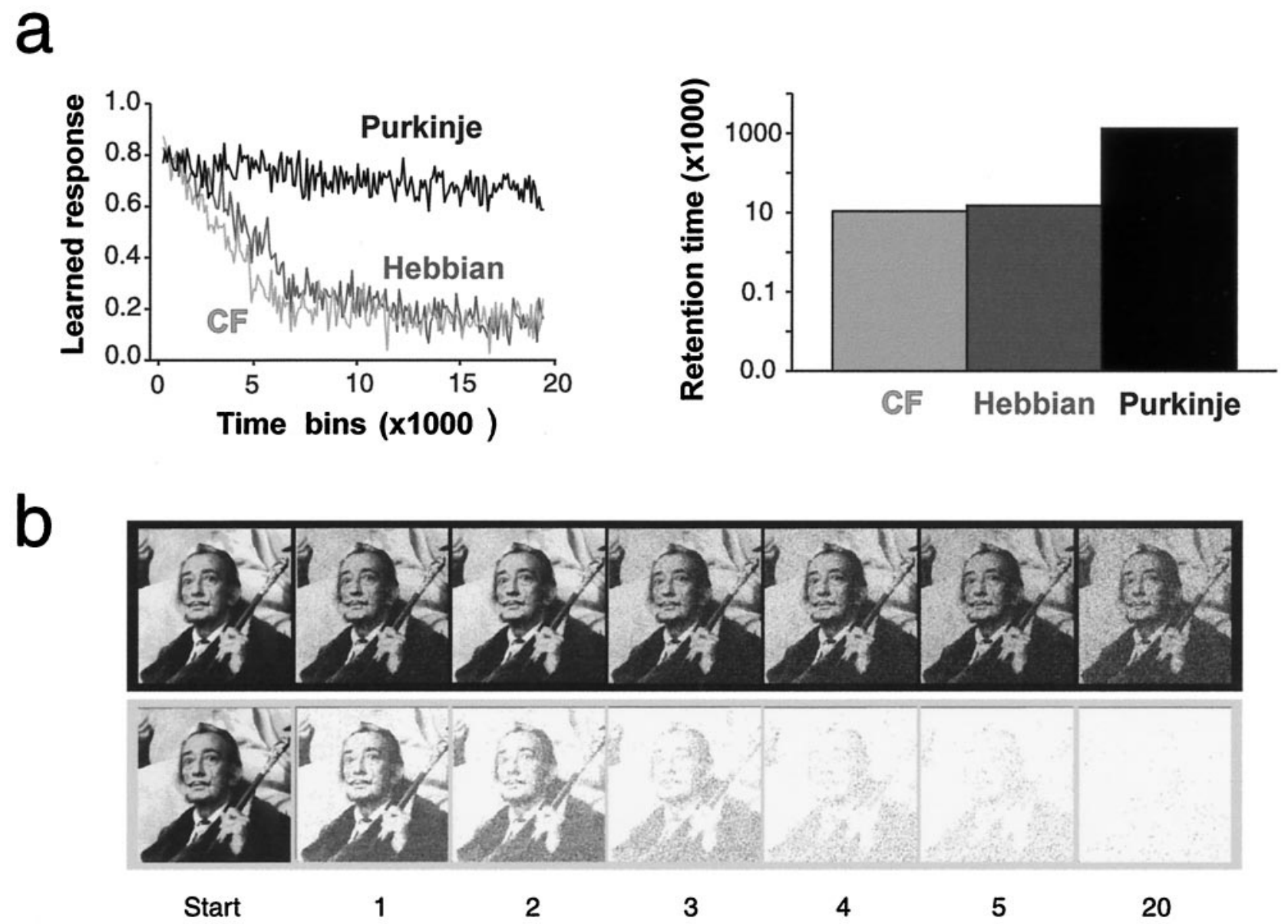

Time bins $(\mathbf{x 1 0 0 0 )}$

Figure 4. Retention of simulated conditioned responses when plasticity rules are operational during background input activity. $a$, The left graph shows that the amplitude of the conditioned response decreases rapidly when either a Hebbian (dark gray line) or climbing fiber-dependent (light gray line) plasticity rule is implemented at $m f \rightarrow n u c$ synapses. In contrast, when a Purkinje-dependent rule is used (black line), increases in nucleus cell activity during presentation of the CS could be observed for much longer periods of time (right graph). $b$, The effects that implementing different rules for plasticity have on the persistence of memory can be further illustrated by assuming that the pattern of strengths at $g r \rightarrow P k j$ synapses corresponds to the picture of Salvador Dali. Light pixels correspond to weak synapses, and dark pixels correspond to strong synapses. Implementing a Purkinje-dependent rule in the cerebellar nucleus drives the system to a state of equilibrium where memories are retained, because although synapses are continually changing (note that the encoded picture slowly degrades with time), they are as likely to increase in strength as they are to decrease (top row). In contrast, Hebbian (data not shown) or climbing fiber-dependent (bottom row) rules produce a spontaneous drift of synaptic strength, which ultimately results in saturation and complete loss of memory.

temperature of a single room with two thermostats. Unless they are set to exactly the same temperature (analogous to severe parameter constraints), one will always try to decrease the temperature of the room (analogous to synaptic strengths spontaneously drifting at one site of plasticity), whereas the other will continuously try to increase room temperature (analogous to synaptic strengths drifting at the other site).

\section{DISCUSSION}

Our results illustrate that a molecular mechanism for persistent expression of synaptic plasticity is not sufficient to explain the enduring retention of memories. Instead, the contribution of synaptic plasticity to memory must be considered in the context of the circuits in which modifiable synapses reside. This is often taken into account when considering the relationship between the induction of plasticity and the acquisition of memories. Debates continue, for example, as to whether the patterns of stimulation that induce LTP or LTD are physiological and actually occur in vivo (Otto et al., 1991; Heynen et al., 1996; De Schutter, 1997; Mauk et al., 1997). Our results extend this thinking by highlighting the importance of the interaction between circuits and synapses in the stability of synaptic strengths and the persistence of memories.

The main prediction of our results is that when the background activity of the cerebellar network is considered, only one of three seemingly plausible forms of plasticity in the cerebellar nucleus interacts with network properties to produce learning. Although simulations with Hebbian and climbing fiber-dependent rules could learn in the absence of background inputs, under more realistic conditions their inherent tendency to produce spontaneous drift in synaptic weights precluded their ability to learn and to retain responses. In contrast, Purkinje-controlled plasticity at $m f \rightarrow n u c$ synapses in the cerebellar nucleus appeared to promote an equilibrium of activity that prevented spontaneous drift of synaptic weights and permitted both learning and retention, independent of the amount of background input. Importantly, the 


\section{Purkinje-dependent rule at $m f-n u c$ synapses}

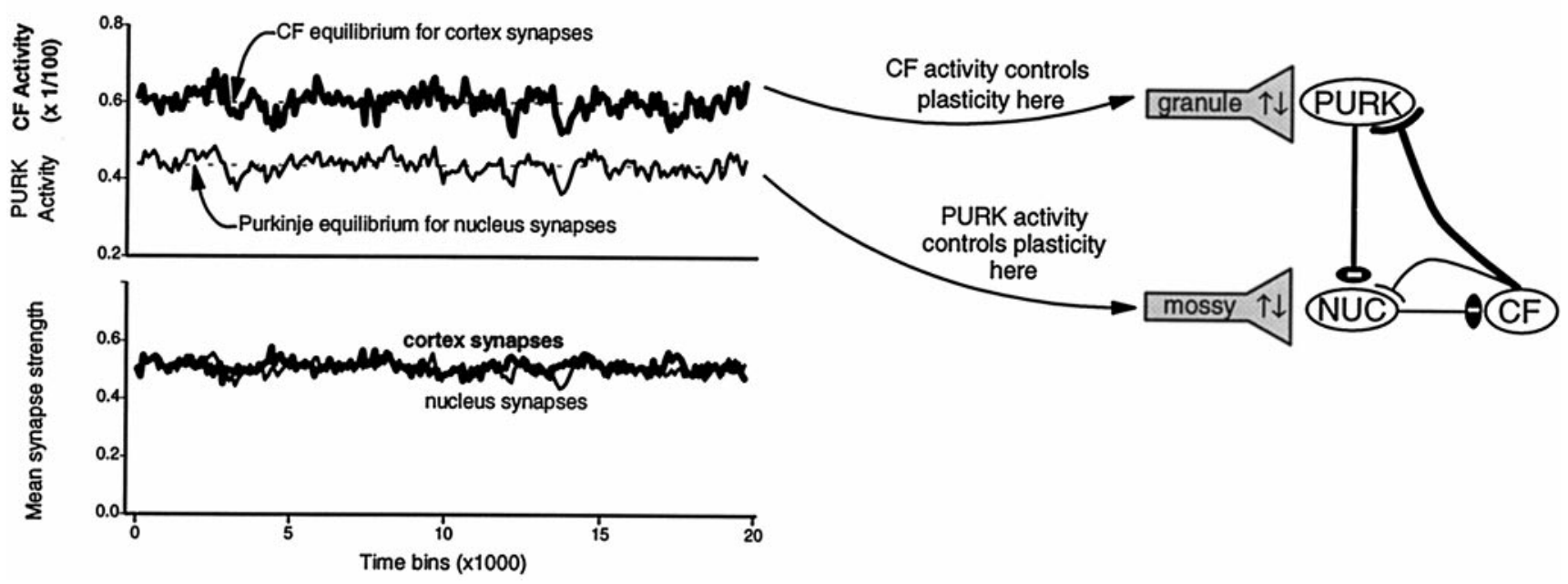

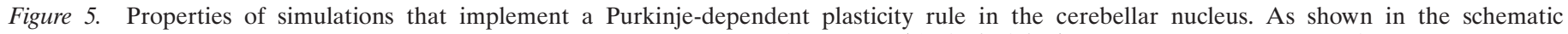

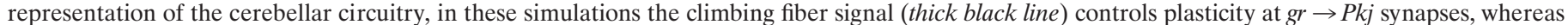

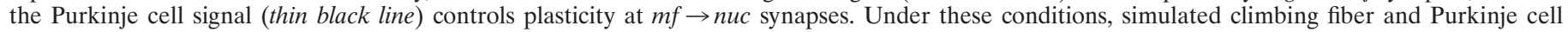

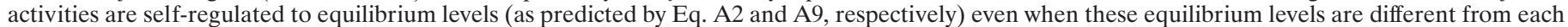

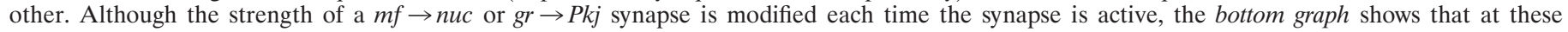
equilibrium levels synapses are as likely to increase as they are to decrease in strength.

ability of this rule to prevent synaptic weight drift did not preclude further learning because conditioned responses could still be extinguished when the CS was presented in the absence of the US.

The potential biological relevance of these data is enhanced by observations that the results are not peculiar to a particular set of parameters. The connectivity of the simulations reflects fundamental systems-level features of the cerebellum and therefore does not depend on exact specification of a large number of parameters. Still, it was necessary to stipulate values for a few relatively free parameters such as the magnitudes of change for LTP and LTD events and the rates of background synaptic activity. However, reducing the model to its critical essentials shows that our results do not vary significantly over a comprehensive range of values for these parameters as long as three fundamental constraints are satisfied: (1) $g r \rightarrow P k j$ synapses in the cerebellar cortex and $m f \rightarrow n u c$ synapses in the cerebellar nucleus are bidirectionally modifiable with LTP and LTD being able to reverse the effects of each other, (2) in addition to being active during learning, these synapses can also be active during nonlearning periods providing further opportunities to modify their strength, and (3) connections in the cerebellar-olivary system are topographically organized in a loop, such that each Purkinje cell influences (via projections to the nucleus) its own climbing fiber input. This observation is consistent with mathematical analyses suggesting that the equilibrium produced by climbing fiberdependent plasticity in the cortex combined with Purkinjedependent plasticity in the nucleus is parameter independent (Kenyon and Mauk, 1994). Similarly, we find that the spontaneous drift of synaptic weights that occurred with Hebbian or climbing fiber-controlled plasticity in the nucleus was an intrinsic and robust property that arises from the interaction between those forms of plasticity and the connectivity of the network. For these unstable rules there exists only one set of parameters where spontaneous drift is decreased significantly, but even in this case a second form of instability operates and eventually causes satu- ration of synaptic weights (data not shown). This robustness shows that neither our results nor their implications depend on tweaking of free parameters, but rather reflect basic characteristics that emerge from interactions between cerebellar connectivity and particular forms of plasticity at the two sites.

Although the simulations relate to cerebellar-mediated motor learning, the implications of our results are not specific to cerebellar synapses. A memory, for example, might be encoded by the induction of LTP at a pattern of hippocampal or neocortical synapses. Any unchecked tendency for systematic drift in synaptic strengths would saturate all synapses at their maximum or minimum value, erasing the pattern of strengths and destroying the memory. As an example of a potential instability inherent in Hebbian plasticity, the strengthened synapses might increase postsynaptic activity and lead to further induction of LTP at other synapses onto the same postsynaptic cell (Sejnowski and Tesauro, 1989; Brown et al., 1990). Recent evidence suggests that intrinsic inhibitory circuitry may help prevent such runaway changes in a number of systems (Artola and Singer, 1987; Steele, Mauk, 1998). Regardless of such details, our results further illustrate how mismatches between the properties of plasticity and the properties of circuits can erase memories despite the existence of molecular mechanisms that are otherwise capable of long-term expression of plasticity.

For the cerebellum, our results suggest that the stability of synaptic weights results from a self-regulating equilibrium of climbing fiber activity controlling LTP/LTD in the cortex and a similar equilibrium of Purkinje activity controlling LTP/LTD in the nucleus. For climbing fibers, this equilibrium depends on circuitry that allows the Purkinje cell to regulate the activity of its single climbing fiber input (Kenyon et al., 1998). In addition to the extensive anatomical tracing investigations that support this precision of connectivity (Voogd and Bigare, 1980; Buisseret-Delmas and Angaut, 1993; Ruigrok, 1997; Voogd and Glickstein, 1998), recent recording studies in primates provide more direct evi- 


\section{Climbing fiber-dependent rule at mf-nuc synapses}

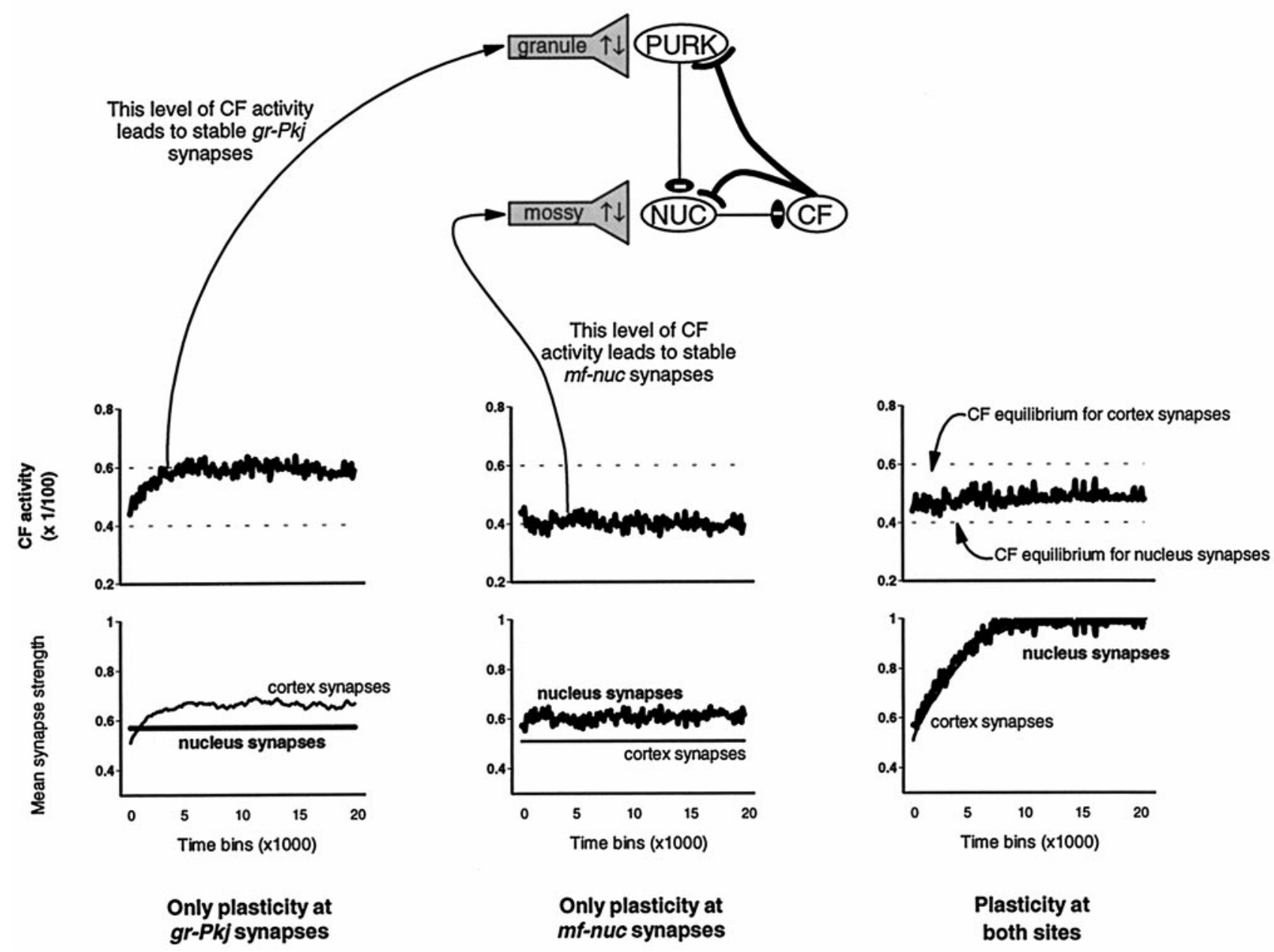

Figure 6. Properties of simulations that implement Hebbian or climbing fiber-dependent plasticity rules in the cerebellar nucleus. As shown in the schematic representation of the cerebellum, simulations that implement a climbing fiber-dependent or Hebbian rule in the nucleus automatically place both $g r \rightarrow P k j$ and $m f \rightarrow n u c$ synapses under the control of a single signal related to the activity of the climbing fiber (thick black line). For the parameters used in these simulations, the level of climbing fiber activity required for equilibrium of $g r \rightarrow$ Pkj synapses is shown in the left column. The top graph in the left column shows that in simulations with plasticity at $m f \rightarrow n u c$ synapses turned off, climbing fiber activity was self-regulated to the level predicted by Equation A2, and that at this equilibrium level the mean strength of $g r \rightarrow P k j$ synapses remained constant (left column, bottom graph). Conversely, the level of simulated climbing fiber activity required for equilibrium of $m f \rightarrow$ nuc synapses is shown in the middle column. The top graph in the middle column shows that in simulations with plasticity at $g r \rightarrow P k j$ synapses turned off, climbing fiber activity was self-regulated to the level predicted by Equation A7, and that at this equilibrium level the mean strength of $m f \rightarrow n u c$ synapses remained constant (middle column, bottom graph). However, as shown in the right column, in simulations with plasticity turned on at both sites, climbing fiber activity fell between these two equilibrium levels (top graph), such that both sets of synapses drifted (bottom graph). Spontaneous drift is reduced only in simulations that use the single set of parameters that makes these two equilibrium levels (Eq. A2 and A7) equal to each other (data not shown).

dence that Purkinje cell activity can calibrate the activity of its own climbing fiber input (Miall et al., 1998). Miall et al. (1998) showed a small but significant relationship between increased Purkinje activity and subsequent increases in the activity of its climbing fiber input, which would presumably induce LTD and drive back down the activities of the Purkinje cell and climbing fiber. Our results and previous mathematical analyses (Kenyon et al., 1998) suggest how such self-regulation of climbing fiber activity could combine with plasticity in the cerebellar cortex to maintain a background equilibrium level of climbing fiber activity where the effects of any LTP and LTD are in balance. The present results extend this concept to Purkinje cell control of plasticity in the cerebellar nucleus. With this rule, plasticity at the two sites is controlled by two different signals, each of which can be at their equilibrium level. However, with the rules found to be unstable, there is in each case a single signal (climber fiber activity) that controls plasticity at both sites. The resulting climbing fiber activity reflects a compromise between the equilibrium of activity needed at each site, producing spontaneous drift of strength at both sets of synapses.

Although the controlling signals and underlying mechanisms of plasticity in the cerebellar nuclei have not been demonstrated explicitly, the implications of the present results are consistent both with existing theory and data from two forms of cerebellarmediated motor learning. In general, analysis of Pavlovian eyelid conditioning and adaptation of the VOR have yielded concordant 
ideas regarding cerebellar mechanisms of learning (Raymond et al., 1996). For both behavioral paradigms, lesion studies implicate plasticity in the cerebellar cortex and nucleus and suggest that lesions of the cerebellar cortex block the induction of plasticity in the cerebellar nuclei (Robinson, 1976; Perrett and Mauk, 1995; Raymond et al., 1996; Mauk, 1997). To explain adaptation of the VOR, Miles and Lisberger (1981; Lisberger, 1994) proposed the hypothesis that plasticity in the cerebellar nucleus contributes to this form of motor learning and that its induction is controlled by inputs from the Purkinje cells. The striking similarity in results from these two different behavioral systems suggests that the mechanism implied is not specific to eyelid conditioning or VOR adaptation but rather is a general feature of cerebellar processing.

The findings of Llinas and Muhlethaler (1988) suggest a concrete but speculative mechanism for inducing LTP/LTD at $m f \rightarrow n u c$ synapses that is consistent with Purkinje cell control of plasticity at this site. Recordings from cerebellar nucleus cells in vitro have revealed a calcium conductance whose activation required depolarization from a hyperpolarized state, rather than depolarization from the resting potential (Llinas and Muhlethaler, 1988). Given that Purkinje cells are normally active at high spontaneous rates, it seems possible that this calcium conductance could be activated during transient decreases in the ongoing inhibitory input that nucleus cells receive from Purkinje cells. Drawing parallels with LTP and LTD in the hippocampus and neocortex (Artola et al., 1990; Mulkey and Malenka, 1992; Dudek and Bear, 1993), the level of $\mathrm{Ca}^{2+}$ in the postsynaptic nucleus cell may be an important factor in determining whether active synapses undergo LTP or LTD. Thus, $m f \rightarrow n u c$ synapses may increase in strength when coactive during the high levels of calcium likely to exist during transient decreases in Purkinje cell activity and decrease in strength when active during lower levels of calcium, as may occur during strong inhibitory input from Purkinje cells.

Although a great deal of effort has been applied to the analysis of the molecular basis of persistent expression of synaptic plasticity, the present results highlight the parallel importance of understanding how these mechanisms are influenced by the networks that provide their inputs. Our results do not obviate the importance or need to study molecular mechanisms of persistent expression. Rather, they suggest the additional challenge of understanding how interactions between these plasticity mechanisms and the properties of the circuits permit induction of plasticity when learning occurs and prevent net changes otherwise. The cerebellum provides a clear example. The degree to which the anatomy and physiology of the cerebellum is known has inspired many models of how it mediates motor learning (Fujita, 1982; Moore et al., 1989; Gluck et al., 1990; Fiala et al., 1996). However, we are not aware of a cerebellar model or simulation that takes into account how synaptic strength remains constant when synapses are activated after movements have been learned. We suggest that any realistic attempt to understand or to model learning in the brain, and specifically motor learning in the cerebellum, must tackle the challenge of ongoing synapse activity and its implications for the induction of unwanted activitydependent plasticity.

\section{APPENDIX \\ Mathematical analysis of plasticity rules at $m \boldsymbol{m} \rightarrow$ nuc synapses}

The intuitive differences between the three rules for plasticity at $m f \rightarrow n u c$ synapses described above can be formalized by considering the expected change in synaptic strength on any given time step. For $g r \rightarrow P k j$ synapses in the cerebellar cortex, an expression for this expected change can be obtained by combining the conditions that lead to LTD with those that lead to LTP:

$$
\Delta w_{i}^{g r}=\frac{\delta_{-}^{g r} \cdot P_{i}^{g r} \cdot P^{c f}}{L T D}+\frac{\delta_{+}^{g r} \cdot P_{i}^{g r} \cdot\left(1-P^{c f}\right)}{L T P} .
$$

This first term in the equation simply formalizes the well characterized rule for LTD observed at $g r \rightarrow P k j$ synapses in the cerebellar cortex. Thus, a $g r \rightarrow P k j$ synapse is expected to undergo LTD by an amount equal to $\delta_{-}^{g r}$ when both its probability of being active, $P_{i}^{g r}$, and the probability of a climbing fiber input, $P^{c f}$, are high. The second term implements LTP by increasing the weight of active $g r \rightarrow P k j$ synapses by $\delta_{+}^{g r}$ when the probability of a climbing fiber input is low [i.e., when $\left(1-P^{c f}\right)$ is high.]

As originally reported by Kenyon et al. (1998), setting this equation to zero yields an expression for the equilibrium level of climbing fiber activity at which the expected change in $g r \rightarrow P k j$ synaptic strength is zero. That is:

$$
\text { when } P^{c f}=\frac{\delta_{+}^{g r}}{\delta_{+}^{g r}-\delta_{-}^{g r}}, \quad \text { then } \Delta w_{i}^{g r}=0 \text {. }
$$

The fundamental characteristic that ensures the stability of the system is that if climbing fiber activity were different from the level expressed by Equation A2, changes would take place at $g r \rightarrow P k j$ synapses that would bring climbing fiber activity back to this equilibrium value. For example, higher than equilibrium levels of climbing fiber activity would result in the induction of LTD at active $g r \rightarrow P k j$ synapses. This would reduce the activity of the Purkinje cells, disinhibiting nucleus cells and in turn decreasing the activity of the climbing fiber toward its equilibrium level. Once climbing fiber activity is at this level, the expected change in synaptic strength is zero (as shown in Eq. A2), and the system remains at this equilibrium state.

In the absence of plasticity at $g r \rightarrow P k j$ synapses, a similar analysis for the three rules of plasticity at $m f \rightarrow n u c$ synapses yields the following equilibrium levels of activity for nucleus cells (Hebbian rule), climbing fiber cells (climbing fiber-dependent rule), and Purkinje cells (Purkinje-dependent rule) respectively.

\section{Hebbian rule}

The expected change in the strength of a $m f \rightarrow n u c$ synapse under the control of a Hebbian rule can be formalized by the following equation:

$$
\Delta w_{i}^{m f}=\frac{\delta_{-}^{m f} \cdot P_{i}^{m f} \cdot\left(1-P^{n u c}\right)}{L T D}+\frac{\delta_{+}^{m f} \cdot P_{i}^{m f} \cdot P^{n u c}}{L T P} .
$$

By setting this equation equal to zero, it is possible to find an expression for the equilibrium level of nucleus activity at which the expected change in $m f \rightarrow n u c$ synaptic strength is zero:

$$
\text { when } P^{n u c}=\frac{\delta_{-}^{m f}}{\delta_{-}^{m f}-\delta_{+}^{m f}}, \quad \text { then } \Delta w_{i}^{m f}=0 \text {. }
$$

However, given the direct inhibition of climbing fibers by the nucleus cell, it is possible to express this equilibrium level of nucleus cell activity in terms of climbing fiber activity. From Equation 12 (Materials and Methods): 


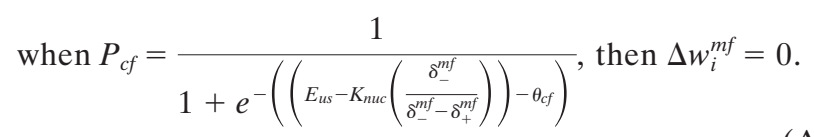

There are two properties that make this Hebbian rule unstable in the context of the cerebellar-olivary circuitry. First, in the absence of plasticity at $g r \rightarrow P k j$ synapses, climbing fiber activity will not be self-regulated to this equilibrium level. For example, higher than equilibrium levels of nucleus activity (Eq. A4) would result in the induction of LTP at active $m f \rightarrow n u c$ synapses. This would increase the activity of the nucleus cell, resulting in the induction of more LTP and further increases in nucleus cell activity. Second, when $g r \rightarrow P k j$ plasticity is added, the activity of the climbing fiber is driven to an intermediate level between the equilibrium level demanded by plasticity at $g r \rightarrow P k j$ synapses (Eq. A2) and that demanded by plasticity at $m f \rightarrow n u c$ synapses (Eq. A5). Yet, this compromise in the level of climbing fiber activity does not fully satisfy either of the equilibrium conditions and ultimately results in the drift of synaptic strengths at both sites of plasticity.

\section{Climbing fiber-dependent rule}

When $m f \rightarrow n u c$ plasticity is under the control of the climbing fiber input to the nucleus, the expected change in synaptic strength and the equilibrium level of climbing fiber activity at which this change is expected to be zero are given by Equations A6 and A7, respectively:

$$
\begin{array}{cc}
\Delta w_{i}^{m f}=\frac{\delta_{-}^{m f} \cdot P_{i}^{m f} \cdot\left(1-P^{c f}\right)}{L T D}+\frac{\delta_{+}^{m f} \cdot P_{i}^{m f} \cdot P^{c f}}{L T P}, \\
\text { when } P^{c f}=\frac{\delta_{-}^{m f}}{\delta_{-}^{m f}-\delta_{+}^{m f}}, & \text { then } \Delta w_{i}^{m f}=0 .
\end{array}
$$

In the absence of plasticity at $g r \rightarrow P k j$ synapses, this climbing fiber-dependent rule for $m f \rightarrow n u c$ synapses is stable by itself. That is, changes will take place so that the level of climbing fiber activity is always driven to the equilibrium value expressed in Equation A7. For example, higher than equilibrium levels of climbing fiber activity would result in the induction of LTP at active $m f \rightarrow n u c$ synapses. This would increase the activity of the nucleus cell, inhibiting the climbing fiber and bringing its activity level back to its equilibrium value (Eq. A7). However, when $g r \rightarrow P k j$ plasticity is added, unless Equations A7 and A2 are set to the same exact value, there will be disagreement between the level of climbing fiber activity that maintains $g r \rightarrow P k j$ synapses unchanged (Eq. A2) and that which does the same for $m f \rightarrow n u c$ synapses (Eq. A7). As it happened when Hebbian control of $m f \rightarrow n u c$ synapses was implemented, the result is that climbing fiber activity is driven to a "compromise" level that in reality does not satisfy either equilibrium condition, leading to the drift of synaptic strengths at both plasticity sites.

\section{Purkinje-dependent rule}

When $m f \rightarrow n u c$ plasticity is under the control of the inhibitory Purkinje input to the nucleus, the expected change in synaptic strength and the equilibrium level of Purkinje cell activity at which this change is expected to be zero are given by Equations A8 and A9, respectively:

$$
\Delta w_{i}^{m f}=\frac{\delta_{-}^{m f} \cdot P_{i}^{m f} \cdot P^{P k j}}{L T D}+\frac{\delta_{+}^{m f} \cdot P_{i}^{m f} \cdot\left(1-P^{P k j}\right)}{L T P},
$$

$$
\text { when } P^{P k j}=\frac{\delta_{+}^{m f}}{\delta_{+}^{m f}-\delta_{-}^{m f}}, \quad \text { then } \Delta w_{i}^{m f}=0 \text {. }
$$

Unlike in the cases for Hebbian or climbing fiber control of nucleus plasticity, this equilibrium condition for $m f \rightarrow n u c$ plasticity can always be satisfied while simultaneously satisfying the equilibrium condition for $g r \rightarrow P k j$ synapses (Eq. A2). This is because the strengths of $g r \rightarrow P k j$ and $m f \rightarrow n u c$ synapses can be dialed up or down until the activities of the Purkinje cell and climbing fiber, respectively, are at the levels demanded by Equations A9 and A2. This ability to regulate Purkinje cell activity by modifying the strengths of $g r \rightarrow P k j$ synapses while regulating climbing fiber activity by modifying $m f \rightarrow n u c$ synaptic strengths allows the system to reach an equilibrium state that prevents synaptic drift by maintaining mean synaptic strength unchanged.

\section{REFERENCES}

Armstrong DM, Rawson JA (1979) Activity patterns of cerebellar cortical neurones and climbing fibre afferents in the awake cat. J Physiol (Lond) 289:425-448.

Artola A, Singer W (1987) Long-term potentiation and NMDA receptors in rat visual cortex. Nature 330:649-652.

Artola A, Singer W (1993) Long-term depression of excitatory synaptic transmission and its relationship to long-term potentiation. Trends Neurosci 16:480-487.

Artola A, Brocher S, Singer W (1990) Different voltage-dependent thresholds for inducing long-term depression and long-term potentiation in slices of rat visual cortex. Nature 347:69-72.

Bear MF, Malenka RC (1994) Synaptic plasticity: LTP and LTD. Curr Opin Neurobiol 4:389-399.

Bell CC, Han VZ, Sugawara Y, Grant K (1997) Synaptic plasticity in a cerebellum-like structure depends on temporal order. Nature 387:278-281.

Brown TH, Kairiss EW, Keenan CL (1990) Hebbian synapses: biophysical mechanisms and algorithms. Annu Rev Neurosci 13:475-511.

Buisseret-Delmas C, Angaut P (1993) The cerebellar olivo-corticonuclear connections in the rat. Prog Neurobiol 40:63-87.

Chen C, Thompson RF (1995) Temporal specificity of long-term depression in parallel fiber-Purkinje synapses in rat cerebellar slice. Learning Memory 2:185-198.

Chen WR, Lee S, Kato K, Spencer DD, Shepherd GM, Williamson A (1996) Long-term modifications of synaptic efficacy in the human inferior and middle temporal cortex. Proc Natl Acad Sci USA 93:8011-8015.

Cull-Candy SG, Brickley SG, Misra C, Feldmeyer D, Momiyama A, Farrant M (1998) NMDA receptor diversity in the cerebellum: identification of subunits contributing to functional receptors. Neuropharmacology 37:1369-1380.

De Schutter E (1997) A new functional role for cerebellar long-term depression. Prog Brain Res 114:529-542.

Dudek SM, Bear MF (1993) Bidirectional long-term modification of synaptic effectiveness in the adult and immature hippocampus. J Neurosci 13:2910-2918.

Eccles JC, Ito M, Szentágothai J (1967) The cerebellum as a neuronal machine. New York: Springer-Verlag.

Eccles JC, Faber DS, Murphy JT, Sabah NH, Taborikova H (1971) Afferent volleys in limb nerves influencing impulse discharges in cerebellar cortex. I. In mossy fibers and granule cells. Exp Brain Res 13:15-35.

Ekerot CF, Kano M (1989) Stimulation parameters influencing climbing fibre induced long-term depression of parallel fibre synapses. Neurosci Res 6:264-268.

Fiala JC, Grossberg S, Bullock D (1996) Metabotropic glutamate receptor activation in cerebellar Purkinje cells as substrate for adaptive timing of the classically conditioned eye-blink response. J Neurosci 16:3760-3774.

Fujita M (1982) Adaptive filter model of the cerebellum. Biol Cybern 45:195-206.

Gluck MA, Reifsnider ES, Thompson RF (1990) Adaptive signal processing and the cerebellum: models of classical conditioning and VOR 
adaptation. In: Neuroscience and connectionist theory (Gluck MA, Rumelhart DE, eds), pp 131-185. Hillsdale, NJ: Lawrence Erlbaum Associates.

Haykin SS (1994) Neural networks: a comprehensive foundation. New York: Macmillan.

Hess G, Donoghue JP (1996) Long-term depression of horizontal connections in rat motor cortex. Eur J Neurosci 8:658-665.

Heynen AJ, Abraham WC, Bear MF (1996) Bidirectional modification of CA1 synapses in the adult hippocampus in vivo. Nature 381:163-166.

Hirano T (1990) Depression and potentiation of the synaptic transmission between a granule cell and a Purkinje cell in rat cerebellar culture. Neurosci Lett 119:141-144.

Ito M (1984) The cerebellum and neural control. New York: Raven.

Ito M (1989) Long-term depression. Annu Rev Neurosci 12:85-102.

Karachot L, Kado RT, Ito M (1994) Stimulus parameters for induction of long-term depression in in vitro rat Purkinje cells. Neurosci Res 21:161-168.

Keating JG, Thach WT (1995) Nonclock behavior of inferior olive neurons: interspike interval of Purkinje cell complex spike discharge in the awake behaving monkey is random. J Neurophysiol 73:1329-1340.

Kenyon GT, Mauk MD (1994) Cerebellar mechanisms of eyelid conditioning: mathematical analysis of plasticity at two competing sites. Soc Neurosci Abstr 20:1208.

Kenyon GT, Medina JF, Mauk MD (1998) A mathematical model of the cerebellar-olivary system I: self-regulating equilibrium of climbing fiber activity. J Comput Neurosci 5:17-33.

Kirkwood A, Dudek SM, Gold JT, Aizenman CD, Bear MF (1993) Common forms of synaptic plasticity in the hippocampus and neocortex in vitro. Science 260:1518-1521.

Lewis JL, LoTurco JJ, Solomon PR (1987) Lesions of the middle cerebellar peduncle disrupt acquisition and retention of the rabbit's classically conditioned nictitating membrane response. Behav Neurosci 101:151-157.

Linden DJ (1994) Long-term synaptic depression in the mammalian brain. Neuron 12:457-472.

Lisberger SG (1994) Neural basis for motor learning in the vestibuloocular reflex of primates. III. Computational and behavioral analysis of the sites of learning. J Neurophysiol 72:974-998.

Llinas R, Muhlethaler M (1988) An electrophysiological study of the in vitro, perfused brain stem-cerebellum of adult guinea-pig. J Physiol (Lond) 404:215-240.

Mauk MD (1997) Roles of cerebellar cortex and nuclei in motor learning: contradictions or clues? Neuron 18:343-346.

Mauk MD, Donegan NH (1997) A model of Pavlovian eyelid conditioning based on the synaptic organization of the cerebellum. Learning Memory 3:130-158.

Mauk MD, Steinmetz JE, Thompson RF (1986) Classical conditioning using stimulation of the inferior olive as the unconditioned stimulus. Proc Natl Acad Sci USA 83:5349-5353.

Mauk MD, Steele PM, Medina JF (1997) Cerebellar involvement in motor learning. Neuroscientist 3:303-313.

McCormick DA, Thompson RF (1984) Cerebellum: essential involvement in the classically conditioned eyelid response. Science 223:296-299.

McCormick DA, Steinmetz JE, Thompson RF (1985) Lesions of the inferior olivary complex cause extinction of the classically conditioned eyeblink response. Brain Res 359:120-130.

Miall RC, Keating JG, Manil J, Thach WT (1998) Simple spike activity predicts occurrence of complex spikes in cerebellar Purkinje cells. Nature Neurosci 1:13-15.

Miles FA, Lisberger SG (1981) Plasticity in the vestibulo-ocular reflex: a new hypothesis. Annu Rev Neurosci 4:273-299.

Moore JW, Desmond JE, Berthier NE (1989) Adaptively timed conditioned responses and the cerebellum: a neural network approach. Biol Cybern 62:17-28.

Mulkey RM, Malenka RC (1992) Mechanisms underlying induction of homosynaptic long-term depression in area CA1 of the hippocampus. Neuron 9:967-975.

Otto T, Eichenbaum H, Wiener SI, Wible CG (1991) Learning-related patterns of CA1 spike trains parallel stimulation parameters optimal for inducing hippocampal long-term potentiation. Hippocampus 1:181-192.
Pavlov IP (1927) Conditioned reflexes: an investigation of the physiological activity of the cerebral cortex. London: Oxford UP.

Perrett SP, Mauk MD (1995) Extinction of conditioned eyelid responses requires the anterior lobe of cerebellar cortex. J Neurosci 15:2074-2080.

Perrett SP, Ruiz BP, Mauk MD (1993) Cerebellar cortex lesions disrupt learning-dependent timing of conditioned eyelid responses. J Neurosci 13:1708-1718.

Racine RJ, Wilson DA, Gingell R, Sunderland D (1986) Long-term potentiation in the interpositus and vestibular nuclei in the rat. Exp Brain Res 63:158-162.

Raymond JL, Lisberger SG, Mauk MD (1996) The cerebellum: a neuronal learning machine? Science 272:1126-1131.

Robinson DA (1976) Adaptive gain control of vestibuloocular reflex by the cerebellum. J Neurophysiol 39:954-969.

Ruigrok TJ (1997) Cerebellar nuclei: the olivary connection. In: The cerebellum: from structure to control (de Zeeuw CI, Strata P, Voogd J, eds), pp 167-192. Amsterdam: Elsevier.

Sakurai M (1987) Synaptic modification of parallel fibre-Purkinje cell transmission in in vitro guinea-pig cerebellar slices. J Physiol (Lond) 394:463-480.

Salin PA, Malenka RC, Nicoll RA (1996) Cyclic AMP mediates a presynaptic form of LTP at cerebellar parallel fiber synapses. Neuron 16:797-803.

Schneiderman N, Fuentes I, Gormezano I (1962) Acquisition and extinction of the classically conditioned eyelid response in the albino rabbit. Science 136:650-652.

Sejnowski TJ (1977) Statistical constraints on synaptic plasticity. J Theor Biol 69:385-389.

Sejnowski TJ, Tesauro G (1989) The Hebb rule for synaptic plasticity: algorithms and implementations. In: Neural models of plasticity: experimental and theoretical approaches (Byrne JH, Berry WO, eds), pp 94-103. San Diego: Academic.

Shibuki K, Okada D (1992) Cerebellar long-term potentiation under suppressed postsynaptic $\mathrm{Ca}^{2+}$ activity. NeuroReport 3:231-234.

Siegelbaum SA, Kandel ER (1991) Learning-related synaptic plasticity: LTP and LTD. Curr Opin Neurobiol 1:113-120.

Solomon PR, Lewis JL, LoTurco JJ, Steinmetz JE, Thompson RF (1986) The role of the middle cerebellar peduncle in acquisition and retention of the rabbit's classically conditioned nictitating membrane response. Bull Psychon Soc 24:74-78.

Steele PM, Mauk MD (1999) Inhibitory control of LTP and LTD: stability of synapse strength. J Neurophysiol 81:1559-1566.

Steinmetz JE, Lavond DG, Thompson RF (1985) Classical conditioning of the rabbit eyelid response with mossy fiber stimulation as the conditioned stimulus. Bull Psychon Soc 28:245-248.

Steinmetz JE, Rosen DJ, Chapman PF, Lavond DG, Thompson RF (1986) Classical conditioning of the rabbit eyelid response with a mossy-fiber stimulation CS: I. Pontine nuclei and middle cerebellar peduncle stimulation. Behav Neurosci 100:878-887.

Steinmetz JE, Logan CG, Rosen DJ, Thompson JK, Lavond DG, Thompson RF (1987) Initial localization of the acoustic conditioned stimulus projection system to the cerebellum essential for classical eyelid conditioning. Proc Natl Acad Sci USA 84:3531-3535.

Steinmetz JE, Logan CG, Thompson RF (1988) Essential involvement of mossy fibers in projecting the conditioned stimulus to the cerebellum during classical conditioning. In: Cellular mechanisms of conditioning and behavioral plasticity (Woody DL, Alkon DL, McGaugh JL, eds), pp 143-148. New York: Plenum.

Thach WT (1968) Discharge of Purkinje and cerebellar nuclear neurons during rapidly alternating arm movements in the monkey. J Neurophysiol 31:785-797.

Thompson RF (1986) The neurobiology of learning and memory. Science 233:941-947.

Thompson RF, Krupa DJ (1994) Organization of memory traces in the mammalian brain. Annu Rev Neurosci 17:519-549.

Voogd J, Bigare F (1980) Topographical distribution of olivary and cortico nuclear fibers in the cerebellum: a review. In: The inferior olivary nucleus: anatomy and physiology (Courville J, Université M, eds), pp 207-234. New York: Raven.

Voogd J, Glickstein M (1998) The anatomy of the cerebellum. Trends Neurosci 21:370-375. 